\title{
Comparison of frequency-domain and continuous-wave near-infrared spectroscopy devices during the immediate transition
}

\author{
Tanja van Essen ${ }^{1 *} \mathbb{D}$, Tom G. Goos ${ }^{1,2}$, Liza van Ballegooijen', Gerhard Pichler ${ }^{3,4}$, Berndt Urlesberger ${ }^{3,4}$,
} Irwin K. M. Reiss ${ }^{1}$ and Rogier C. J. de Jonge ${ }^{1,5^{*}}$

\begin{abstract}
Background: Non-invasive monitoring of cerebral tissue oxygen saturation $\left(\mathrm{rCSO}_{2}\right)$ during transition is of growing interest. Different near-infrared spectroscopy (NIRS) techniques have been developed to measure $\mathrm{rCSO}_{2}$. We compared $\mathrm{rCSO}_{2}$ values during the immediate transition in preterm neonates measured with frequency-domain NIRS (FD-NIRS) with those measured with continuous-wave NIRS (CW-NIRS) devices in prospective observational studies.

Methods: We compared $\mathrm{rCSO}_{2}$ values measured with an FD-NIRS device during the first 15 min after birth in neonates with a gestational age $\geq 30$ weeks but $<37$ weeks born at the Erasmus MC- Sophia Children's Hospital, Rotterdam, the Netherlands, with similar values measured with a CW-NIRS device in neonates born at the Medical University of Graz, Austria. Mixed models were used to adjust for repeated $\mathrm{rCSO}_{2}$ measurements, with fixed effects for time (non-linear), device, respiratory support and the interaction of device and respiratory support with time. Additionally, parameters such as total haemoglobin concentration and oxygenated and deoxygenated haemoglobin concentrations measured by FD-NIRS were analysed.

Results: Thirty-eight FD-NIRS measurements were compared with $58 \mathrm{CW}$-NIRS measurements. The FD-NIRS $\mathrm{rCSO}_{2}$ values were consistently higher than the CW-NIRS $\mathrm{rCSO}_{2}$ values in the first $12 \mathrm{~min}$, irrespective of respiratory support. After adjustment for respiratory support, the time-dependent trend in $\mathrm{rCSO}_{2}$ differed significantly between techniques $(p<0.01)$.

Conclusion: As cerebral saturation measured with the FD-NIRS device differed significantly from that measured with the CW-NIRS device, differences in absolute values need to be interpreted with care. Although FD-NIRS devices have technical advantages over CW-NIRS devices, FD-NIRS devices may overestimate true cerebral oxygenation and their benefits might not outweigh the usability of the more clinically viable CW-NIRS devices.
\end{abstract}

Keywords: Near-infrared spectroscopy, Continuous-wave, Frequency-domain, Transition, Preterm neonate

\section{Background}

Poor cerebral perfusion and fluctuations in cerebral oxygenation can adversely affect brain development [1-3]. Regional tissue oxygenation can be continuously monitored with near-infrared spectroscopy (NIRS). The use of this non-invasive technique in neonates allows for the

\footnotetext{
* Correspondence: t.vanessen@erasmusmc.nl; r.c.j.dejonge@erasmusmc.nl ${ }^{1}$ Department of Pediatrics, Division of Neonatology, Erasmus MC - Sophia Children's Hospital, Dr. Molewaterplein 40, 3015GD Rotterdam, The Netherlands

Full list of author information is available at the end of the article
}

deployment of interventions to stabilize or improve cerebral oxygenation and perfusion [1, 4]. Various NIRS-based measurement techniques, devices and sensors have been developed to monitor cerebral oxygenation. Most of the clinically used NIRS devices make use of continuous-wave (CW) light sources, which emit light with a constant intensity (Fig. 1a). CW-NIRS calculates the oxygen saturation from the measured absorption without the possibility of calculating the absolute oxygenated and deoxygenated haemoglobin concentrations. This technique has great value in monitoring the dynamics of cerebral tissue 


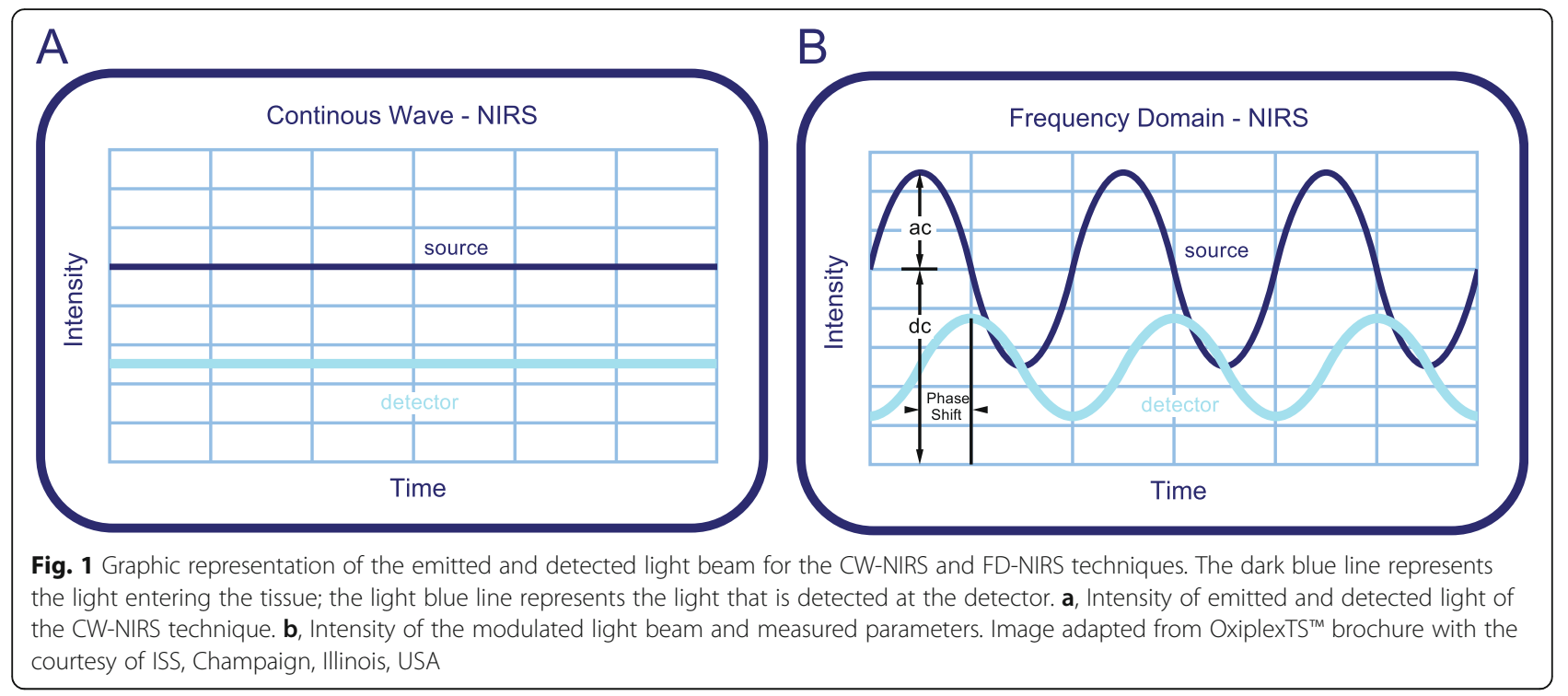

oxygenation but lacks in providing accurate absolute oxygenation estimates [5].

Another NIRS technique, frequency-domain (FD) NIRS, allows for the determination of absolute values of haemoglobin concentration by modulating the intensity of the emitted light by a sinusoidal function. This results in amplitude of modulation (AC), average intensity (DC) and phase-shift (Fig. 1b) measurements, from which the absolute absorption and scattering coefficients are determined. The phase shift represents the delay between the emitted and detected waves of light and changes with the optical path length of the light through the tissue. From the absorption and scattering coefficients, absolute haemoglobin concentrations and subsequently regional cerebral oxygen saturation $\left(\mathrm{rcSO}_{2}\right)$ are calculated. Measurements of absolute concentrations of haemoglobin may improve the interpretation of brain haemodynamics during various clinical settings and allow for withinand between-patient comparisons. While the CW-NIRS technique has been extensively evaluated in the clinical setting, including the transition after birth [6], bedside FD-NIRS devices are not yet available, and the technique has yet to be evaluated properly in a clinical setting.

The aim of the present study was to compare the results of CW-NIRS and FD-NIRS monitoring devices in preterm neonates immediately after birth. If, in theory, both devices reflect true tissue oxygenation, we hypothesized that the cerebral oxygenation values measured with each device would be similar in absolute values as well as in trend during the transition after birth. As cerebral saturation gradually increases during the transition phase after birth, the measurement techniques can be compared over the full dynamic range of tissue oxygenation. In addition, data obtained with the FD-NIRS device might help us to understand the physiological changes that occur immediately after birth.

\section{Methods}

In this study, data from prospective observational studies performed at the Erasmus MC - Sophia Children's Hospital, Rotterdam, the Netherlands, and the Medical University of Graz, Graz, Austria, were compared. Those studies concerned neonates with a gestational age $\geq 30$ weeks but $<37$ weeks, monitored during the first $15 \mathrm{~min}$ of life with an FD-NIRS device in Rotterdam, and with a CW-NIRS device in Graz.

\section{FD-NIRS}

FD-NIRS measurements were performed in newborn neonates between May 2015 and October 2017, delivered either vaginally or by caesarean section. Neonates with suspected congenital or chromosomal anomalies were excluded from the analyses. The local Medical Ethics Review Board waived approval (argument: "Medical Research in Human Subjects Act does not apply to this research proposal"; MEC-2011-415).

FD-NIRS measurements were performed using the OxiplexTS $^{\text {тा }}$ (ISS, Inc., Champaign, IL, USA) with the Infant Flexible Sensor, containing one detector and four emitter positions with two emitters each (eight in total). Emitter-detector distances on this device range from 1.5 $\mathrm{cm}$ to $4.0 \mathrm{~cm}$. The device uses near-infrared light at two different wavelengths: $684 \mathrm{~nm}$ and $828 \mathrm{~nm}$. Continuous FD-NIRS measurement data were collected over a maximum of $15 \mathrm{~min}$, with a sampling rate of $0.5 \mathrm{~s}(2 \mathrm{~Hz})$.

In addition to the preductal arterial oxygen saturation $\left(\mathrm{SpO}_{2}\right)$ measurement used as standard of care, postductal $\mathrm{SpO}_{2}$ and heart rate (HR) were measured with pulse oximetry (Masimo Radical-7, Irvine, CA, USA). Any 
respiratory support was provided using a T-piece resuscitator (Neopuff, Fisher \& Paykel Healthcare, Auckland, New Zealand).

\section{CW-NIRS}

For comparison, $\mathrm{rcSO}_{2}$ values obtained with a CW-NIRS device were provided by the Graz University Medical Centre. The data were obtained in prospective observational studies approved by the Regional Committee on Biomedical Research Ethics at the Medical University of Graz (EK- number: 19-291ex07/08, 23-403ex10/11, 27-465ex14/15). Written informed consent was obtained from the parents before the birth of the infant. All included neonates had been delivered by elective caesarean section. The CW-NIRS device used was the INVOS $5100 \mathrm{C}$ Cerebral/Somatic Oximeter (Medtronic, Minneapolis, MN, USA). Selected data have been published previously; methods and data acquisition are described in more detail in these publications $[7,8]$.

\section{FD-NIRS data acquisition}

A sensor calibration procedure was carried out before every measurement. The measurement time started when the umbilical cord was clamped, as this was clinical practice in both centres. Further, in both centres, as per the local protocol at the time the study was conducted, immediate cord clamping was the standard procedure and was performed before $30 \mathrm{~s}$. The FD-NIRS sensor was placed on the baby's left frontotemporal forehead. A pulse oximeter was placed postductally on the baby's left foot. Upon signs of respiratory distress, supplemental oxygen and positive end-expiratory pressure (PEEP) therapy or ventilation were applied according to the local protocol. Physicians were blinded to the $\mathrm{rcSO}_{2}$ and $\mathrm{SpO}_{2}$ measurements. In contrast to the data acquisition method applied in Graz, where all neonates were monitored during the full first $15 \mathrm{~min}$ after birth, in Rotterdam, we were obligated by the Medical Ethics Review Board to stop the measurements once routine medical care had been completed and the baby was stable before the completion of the first $15 \mathrm{~min}$ after birth.

In addition to $\mathrm{rcSO}_{2}$, the FD-NIRS device provides absolute values of the total haemoglobin concentration $(\mathrm{THb})$, oxygenated $\left(\mathrm{O}_{2} \mathrm{Hb}\right)$ and deoxygenated haemoglobin (HHb) concentration. To account for the displacement of the sensor, during postprocessing of the FD-NIRS data, measurements were discarded when the $\mathrm{AC}$ was below 1 , the $\mathrm{DC}$ was below 10 or when values were considered non-physiological (values below 0 or above 100). Afterwards, the data were averaged over six seconds.

\section{Phase shift}

To compare the CW-NIRS device and the FD-NIRS device, we evaluated the effect of the phase shift on the FD-NIRS measurement. Median (interquartile range; IQR) phase shifts are reported, and the effect of the change of phases over time for both wavelengths on $\mathrm{rcSO}_{2}$ was evaluated and presented for a single FD-NIRS measurement. All the phase-shift pairs that occurred during this measurement were used to recalculate a matrix of possible cerebral saturations. The percentiles of these recalculated cerebral saturations for all occurring phase-shift pairs were compared to the original raw cerebral saturation data.

\section{Statistics}

Categorical variables are presented as numbers (\%); continuous variables are presented as medians (IQRs). Demographics were compared using Fisher's exact test for categorical data and the Mann-Whitney $U$ test for continuous data. A per-minute analysis was performed for the $\mathrm{rCSO}_{2}$ values, comparing FD-NIRS and CWNIRS data. The first minute was excluded from the analyses to account for sensor placement. For comparison between devices, data were stratified for the need for respiratory support [7].

To adjust for the repeated $\mathrm{rCSO}_{2}$ measurements, we used mixed models to analyse the course of $\mathrm{rcSO}_{2}$, $\mathrm{SpO}_{2}, \mathrm{THb}, \mathrm{O}_{2} \mathrm{Hb}$, and $\mathrm{HHb}$ over time. The following fixed effects were considered in the model using backwards selection: the need for respiratory support, delivery method, gestational age, whether the neonate was small for gestational age (dichotomous) and sex. For the random effects, the use of random intercept and slopes were evaluated. To take into account non-linearity in the relation between time and $\mathrm{rcSO}_{2}, \mathrm{SpO}_{2}, \mathrm{THb}, \mathrm{O}_{2} \mathrm{Hb}$, and $\mathrm{HHb}$, we explored the use of splines and quadratic terms for time. The final model used a mixed model with fixed effects for (non-linear) time, measurement technique (only for $\mathrm{rcSO}_{2}$ and $\mathrm{SpO}_{2}$ ) and respiratory support and a random intercept and slope as random effects. To account for non-linearity, a natural spline with 2 knots for time fit the data best in all mixed models. The results are presented as effect plots of the estimates and their 95\% confidence intervals. A two-sided $P$-value of $<0.05$ was considered statistically significant. Statistical analyses were performed using the computing environment $\mathrm{R}$ (v3.4.1) [9].

\section{Results}

Of 39 eligible neonates in Rotterdam, thirty-eight were included and measured with FD-NIRS. One neonate was diagnosed with ventriculomegaly and therefore excluded. Data from 58 neonates measured with CW-NIRS were available from the Medical University of Graz. Four 
neonates in Graz had been intubated and were therefore excluded. Table 1 summarizes demographic and clinical data. The groups significantly differed in gestational age and therefore in birthweight and head circumference. After adjustment for gestational age according to Fenton et al. [10], the differences in birthweight and head circumference were not statistically significant. Thirty-four (59\%) neonates from the CW-NIRS group and 25 (66\%) from the FD-NIRS group needed respiratory support during the first $15 \mathrm{~min}$ after birth $(p=0.62)$.

\section{Comparison}

The effect plots of the estimated means stratified for the need for respiratory support are shown for both the CW-NIRS and FD-NIRS measurements (Fig. 2a and b). In the first $12 \mathrm{~min}$, the $\mathrm{rcSO}_{2}$ values for the FD-NIRS device were consistently higher than those for the CWNIRS device. After adjustment for respiratory support, the overall trend of $\mathrm{rCSO}_{2}$ over time differed significantly between the devices $(p<0.01)$. Irrespective of the device used, the need for respiratory support significantly influenced cerebral oxygenation $(p<0.01)$.

\section{Phase shift}

The raw cerebral saturation values of a single measurement are shown in dark blue in Fig. 3a, with percentiles of recalculated cerebral saturation values based on all occurring phase-shift pairs. Additionally, the raw phaseshift values for both wavelengths, $684 \mathrm{~nm}$ (light blue line) and $828 \mathrm{~nm}$ (dark blue line), are shown in Fig. 3b. The increase in the phase shift of the $828 \mathrm{~nm}$ wavelength results in the spread of the cerebral saturation centiles of approximately $20 \%$ saturation, and the changes of the phase over time result in a movement through the centiles (Fig. 3a). The visible drop in cerebral saturation is due to a small movement of the sensor during the measurement. The phase-shift values of the single measurement are comparable to the overall phase shift of the whole FD-NIRS group, $684 \mathrm{~nm} 6.23$ [6.04-6.43], $828 \mathrm{~nm} 7.70$ [7.22-8.07] and $684 \mathrm{~nm} 6.04$ [4.46-6.54], $828 \mathrm{~nm} 7.20$ [5.66-7.81], respectively. The median phase-shift values of all included FD-NIRS measurements are presented in supplementary Table I.

\section{$\mathrm{SpO}_{2}$}

Regarding the whole study population, the postductal $\mathrm{SpO}_{2}$ values increased during the transition period (Fig. 2c and d). The $\mathrm{SpO}_{2}$ values were significantly different between neonates with and without the need for respiratory support $(p<0.01)$ and between the CW-NIRS and FD-NIRS groups $(p<0.01) . \mathrm{SpO}_{2}$ values in the first four minutes after birth were predominantly lower in the FD-NIRS group.

\section{Haemoglobin concentration}

The course of THb over the 15-min window did not significantly differ between neonates with and without respiratory support $(p=0.45)$; the same holds true for $\mathrm{O}_{2} \mathrm{Hb}(p=0.20)$ and $\mathrm{HHb}(p=0.10)$. The effect plots of the estimated means (Fig. 4) show a fairly constant $\mathrm{THb}$ value and a decrease over time for $\mathrm{HHb}$. For $\mathrm{O}_{2} \mathrm{Hb}$, an increase over time is seen in the respiratory support group, but such an increase is less evident in the nonrespiratory support group. A significant time-dependent trend was seen for $\mathrm{HHb}$ values $(p<0.01)$ but not for

Table 1 Demographics and clinical data

\begin{tabular}{|c|c|c|c|}
\hline & CW-NIRS $(n=58)$ & FD-NIRS $(n=38)$ & $P$-value \\
\hline Female sex & $30(52 \%)$ & $14(37 \%)$ & 0.22 \\
\hline Gestational age (weeks) & $34 \% / 7\left[32 \frac{4}{7}-35 \% / 7\right]$ & $32 \frac{2}{7}\left[31^{4} / 7-341 / 7\right]$ & $<0.01$ \\
\hline Birthweight (g) & 2066 [1172-2366] & 1763 [1480-2011] & 0.01 \\
\hline Birthweight for age SDS ${ }^{a}$ & $-0.3[-1.0-0.2]$ & $-0.2[-0.8-0.6]$ & 0.50 \\
\hline Head circumference (cm) & $32.0[31.0-33.0]$ & $29.5[28.0-31.8]$ & $<0.01$ \\
\hline Head circumference for age SDS ${ }^{b}$ & $0.0[0.0-1.0]$ & $0.4[-0.8-0.9]$ & 0.91 \\
\hline Arterial umbilical cord pH & $7.31[7.28-7.34]$ & $7.29[7.26-7.34]$ & 0.39 \\
\hline \multicolumn{4}{|l|}{ Apgar } \\
\hline Minute 1 & $8.5[8-9]$ & $8[7-8]$ & 0.02 \\
\hline Minute 5 & $9[8-10]$ & 9 [8-9] & 0.12 \\
\hline Minute 10 & $10[9-10]$ & $9[9-10]$ & 0.25 \\
\hline Caesarean section & $58(100 \%)$ & $27(71 \%)$ & $<0.01$ \\
\hline Respiratory support & $34(59 \%)$ & $25(66 \%)$ & 0.62 \\
\hline Reanimation ${ }^{1}$ & $2(3 \%)$ & $0(0 \%)$ & 0.39 \\
\hline$S G A^{2}$ & $7(12 \%)$ & $7(18 \%)$ & 0.57 \\
\hline
\end{tabular}

Values are median [IQR] or $\mathrm{n}(\%)$; SGA Small for gestational age. ${ }^{a}$ Birthweight-for-age SDS and ${ }^{\mathrm{b}}$ Head circumference-for-age SDS were calculated according to Fenton et al. (ref) 'unknown in $n=1$ from CW-NIRS group; ${ }^{2}$ unknown in $n=1$ from CW-NIRS group 

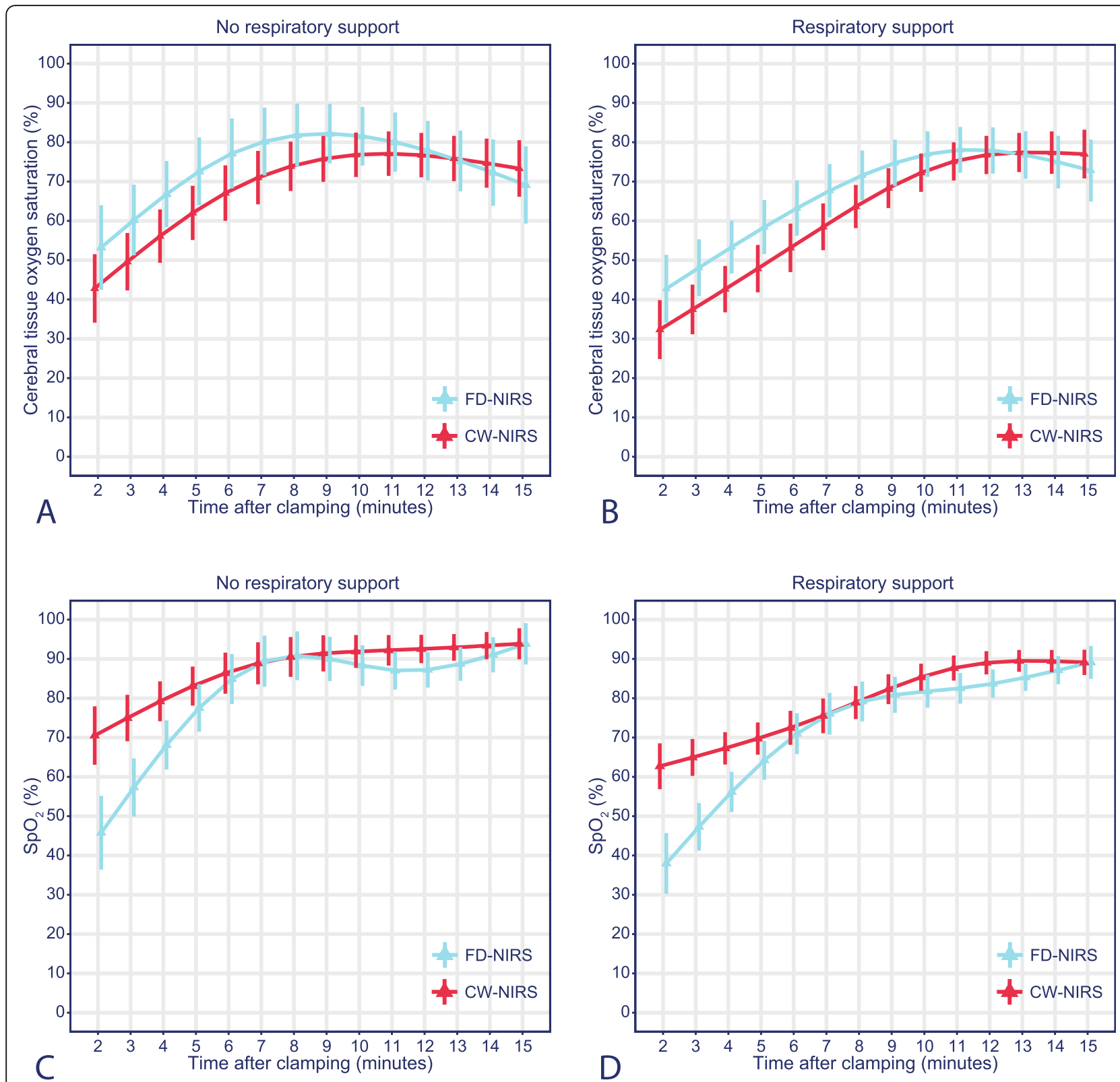

Fig. 2 Effect plots of $\mathrm{rcSO}_{2}$ and $\mathrm{SpO}_{2}$ stratified by the need for respiratory supportSymbols represent the estimated means of $\mathrm{rcSO}_{2}$ and $\mathrm{SpO}_{2}$ with associated $95 \%$ confidence intervals for all difference time points based on a mixed model including (non-linear) time, technique, respiratory support and their interaction with time. $\mathbf{a}$, Effect plot of $\mathrm{rCSO}_{2}$ for neonates not requiring respiratory support. $\mathbf{b}$, Effect plot of $\mathrm{rcSO}_{2}$ for neonates requiring respiratory support. c, Effect plot of $\mathrm{SpO}_{2}$ for neonates not requiring respiratory support. d, Effect plot of $\mathrm{SpO}_{2}$ for neonates requiring respiratory support.

THb values $(p=0.45)$. The time-dependent trend of $\mathrm{O}_{2} \mathrm{Hb}$ did not reach statistical significance $(p=0.08)$.

\section{Discussion}

We compared the outcomes of two different measurement devices of cerebral oxygenation, INVOS 5100C (CW-NIRS) and OxiplexTS ${ }^{\mathrm{Tn}}$ (FD-NIRS), during the immediate transition after birth in preterm neonates. After adjustment for the need for respiratory support, the time- dependent trend of the increase in cerebral oxygenation measured with the FD-NIRS device was significantly different from that measured with the CW-NIRS device. Not only the onset but also the slope differed over time. The need for respiratory support significantly influenced the course of the increase in cerebral saturation, irrespective of the device used.

From a technical perspective, the FD-NIRS device is superior. Clinically, we expect to see low cerebral saturation 


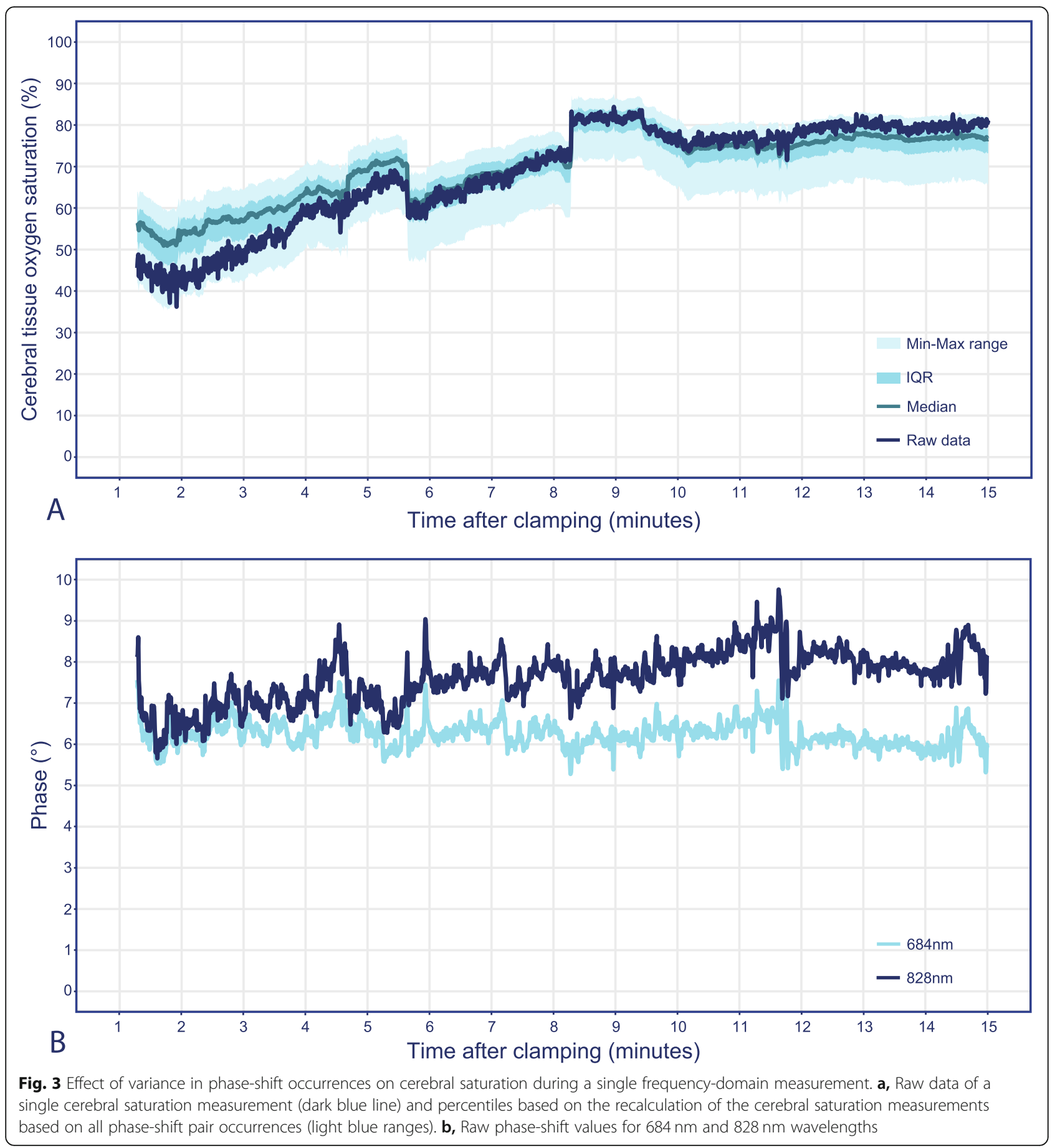

values at the beginning of the transition after birth. However, this is largely because most literature on cerebral saturation during the transition after birth is based on low saturation measurements with the INVOS. The lack of a gold-standard technique for the measurement cerebral saturation during this period makes it difficult to assess if these values are correct. From a medical point of view, we expect the $\mathrm{SpO}_{2}$ values to be higher than the measured $\mathrm{rcSO}_{2}$ values, which is not the case for the FD-NIRS group. This may indicate that FD-NIRS overestimates cerebral saturation.

The phase-shift values of $684 \mathrm{~nm}$ remain fairly constant throughout the single measurement, whereas the phase-shift values of $828 \mathrm{~nm}$ start at roughly the same point as $684 \mathrm{~nm}$ but increase over time. The magnitude of the change in the phase shift directly influences the spread between the centiles of the recalculated cerebral saturations in Fig. 3. Supplementary Table I shows that 

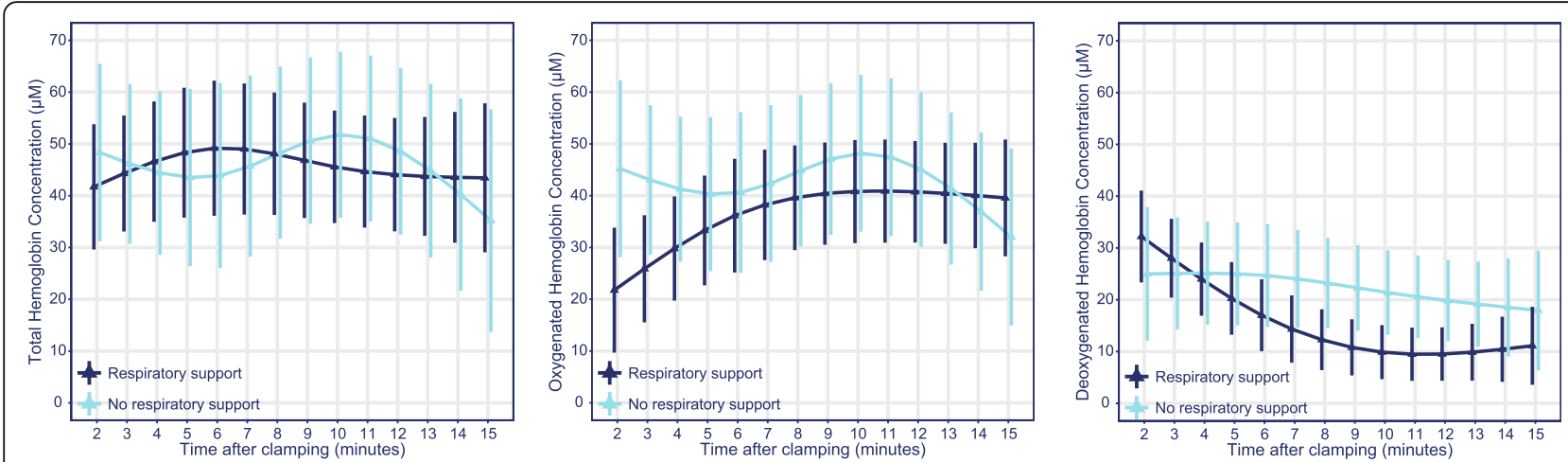

Fig. 4 Effect plots of haemoglobin concentration measurements. a, Total haemoglobin concentration (THb); b, oxygenated haemoglobin concentration $\left(\mathrm{O}_{2} \mathrm{Hb}\right)$; and $\mathbf{c}$, deoxygenated haemoglobin concentration $(\mathrm{HHb})$. THb, $\mathrm{O}_{2} \mathrm{Hb}$ and $\mathrm{HHb}$ are presented as per $\mu \mathrm{M}$. Symbols represent the estimated means with associated $95 \%$ confidence intervals for all difference time points, based on a mixed model including (non-linear) time, respiratory support and their interaction

the presented measurement is comparable to that of most other patients measured with FD-NIRS in this study. The changes in the phase shift over time are likely to improve the accuracy of an FD-NIRS device but limit the comparability between the FD-NIRS device and the CW-NIRS device. Without the knowledge of which physiological parameters are changing when and by how much, or of how they influence the phase measurement over time, and without more research into what other influences on the phase measurement exist, it is impossible to determine if phase-shift correction improves the cerebral saturation measurement. What is clear is that without it, the FD-NIRS measurements could change by as much as $20 \% \mathrm{rcSO}_{2}$.

In both centres, immediate cord clamping was the standard of care. Although not significant, delayed cord clamping (DCC; $>60 \mathrm{~s}$ ) has been shown to be associated with lower $\mathrm{rcSO}_{2}$ immediately after cord clamping but with higher values when stabilized than immediate cord clamping [11]. Additionally, a recent study concluded that $\mathrm{DCC} \geq 30 \mathrm{~s}$ was associated with improved cerebral oxygenation in the first $24 \mathrm{~h}$ after birth [12]. Although immediate cord clamping was the standard of care, an average delay in cord clamping in one of our centres or between infants with and without the need for respiratory support may have contributed to the differences found between devices. Medical professionals should be aware that DCC affects cerebral saturation immediately after birth and may lead to different onset of and increase in $\mathrm{rcSO}_{2}$.

Several studies comparing different devices, sensors and measurement techniques showed differences up to $14 \%$ between $\mathrm{rcSO}_{2}$ values [13-17]. One of these studies made use of stepwise induced hypoxia in healthy volunteers [16]. In line with our findings, measurements of brain-derived parameters in that study significantly differed between FDNIRS and CW-NIRS measurement techniques. FD-NIRS was not considered advantageous in parameter recovery [16]. The within-subject reproducibility of cerebral oxygenation measurements can differ up to $10 \%$ [14, 1719]. Reproducibility, however, is of less importance for trend monitoring. To identify deviations from normal cerebral oxygenation, quantitative monitoring is key [20]. Previous research using FD-NIRS to evaluate infants' brain development showed consistent results from repeated measurements [21, 22]. In 2007, the European Society for Paediatric Research proposed increasing the validity and comparability of peripheral NIRS measurements by standardizing the approach [23]. This important initiative has to be extended to standardizing cerebral NIRS measurements, supported by the possibility of converting values from one oximeter to another. A first valuable attempt has been made using in vitro phantom testing [24, 25]. Furthermore, we recommend that the algorithms used by NIRS devices be published, as this enables us to investigate and understand where the differences in the readings come from.

The $\mathrm{SpO}_{2}$ values of neonates requiring respiratory support were slightly lower than those of neonates who did not require respiratory support. This finding may be due to inadequate lung aeration in neonates requiring respiratory support. Previous studies have reported both lower $\mathrm{SpO}_{2}$ and cerebral oxygen saturation values in neonates requiring respiratory support $[7,26]$. In the present study, the trend in the postductally measured $\mathrm{SpO}_{2}$ values differed between the FD-NIRS and CW-NIRS groups, with predominantly lower values in the FD-NIRS group. Cerebral oxygenation is not solely determined by $\mathrm{SpO}_{2}$, but the higher $\mathrm{rcSO}_{2}$ values in the FD-NIRS group would likely have been even higher if the $\mathrm{SpO}_{2}$ would have been the same for both groups. The difference in $\mathrm{SpO}_{2}$ values between the groups makes proper comparison of the absolute differences between measured $\mathrm{rcSO}_{2}$ values nearly impossible.

The observed THb was stable over the 15-min monitoring period. As changes in arterial haemoglobin concentration are negligible, a clear decrease or increase in cerebral blood volume is not evident from these data, in contrast to 
the findings of Schwaberger et al. [27]. For all three FDNIRS parameters, there were no significant differences between neonates with and without the need for respiratory support. Although not significant, the expected increase over time for $\mathrm{O}_{2} \mathrm{Hb}$ occurred in the respiratory support group but not in the group of neonates who did not receive respiratory support, where even a slight decrease was seen. A possible explanation is the low number of measurements in the latter group, as infants were transferred to the resuscitation table after a few minutes with the parents, motion artefacts occurred and infants not needing respiratory support were more likely to return to the mother before 15 $\mathrm{min}$ as they were considered stable.

The CW-NIRS technique and the FD-NIRS technique each have shortcomings. By using a continuous light source, CW-NIRS assumes a degree of scattering. Due to the modulation of the emitted light, FD-NIRS allows for the quantification of the amount of light scatter, which theoretically results in more accurate measurements of tissue oxygenation. Moreover, FD-NIRS provides highfrequency raw data, resulting in a 'noisier' output. In addition, the device is equipped with a reusable but delicate and cumbersome neonatal sensor that easily results in motion artefacts due to movements of the infant and limits bedside usability and patient comfort. In addition, the OxiplexTS ${ }^{\mathrm{Tw}}$ does not have a CE certificate for clinical use; therefore, it is available only for research purposes. For both devices, tissue homogeneity is assumed, which is debated in regard to the neonatal brain [22, 28]. In an inhomogeneous structure, the use of the mean pathlength by the FD-NIRS may overestimate or underestimate the absolute values of oxygenation.

Several limitations of this study need to be addressed. First, this study compared two devices incorporating different measurement techniques. The observed differences in this study may not be solely attributable to differences between FD-NIRS and CW-NIRS. The differences may result from differences between devices (e.g., different algorithms and emitter-detector differences), as discrepancies between devices using the same measurement technique are reported $[13-15,24,25]$. As this study was a comparison between two specific NIRS devices, the results may not be representative of the whole spectrum of available CW-NIRS and FD-NIRS devices.

Second, FD-NIRS measurement data from our centre were compared with CW-NIRS data from the centre in Graz [8, 29]. Although individual patient data were used, allowing for repeated measurement comparisons using mixed models, a randomized controlled trial would have been preferable. Third, while all included children in Graz were born by caesarean section, the included children in our centre were born either by vaginal delivery or by caesarean section. Nevertheless, previous research showed no differences in $\mathrm{rcSO}_{2}$ with respect to the mode of delivery, nor did our data [30]. Fourth, although not of significant influence on our model, the difference in gestational age between the FD-NIRS and CW-NIRS groups may have caused differences in measured $\mathrm{rcSO}_{2}$ and $\mathrm{SpO}_{2}$ values and may have affected the need for respiratory support. Fifth, the $\mathrm{SpO}_{2}$ was measured postductally, as preductal measurements could not be recorded without interfering with routine medical care in the FD-NIRS patients. As postductal $\mathrm{SpO}_{2}$ values tend to be lower than preductal $\mathrm{SpO}_{2}$ values, fractional tissue oxygen extraction values were not calculated.

\section{Conclusion}

This research demonstrated that $\mathrm{rcSO}_{2}$ values and trends over time differed between devices, mostly in the first minutes after birth. It remains challenging to compare results from different devices, as not only the specific technique but also the specific algorithms and emitterdetector distances may have had a large impact on the outcomes. The FD-NIRS technique might be technically superior to the CW-NIRS technique, but from a clinical point of view, the FD-NIRS technique seems to overestimate true cerebral oxygenation. To date, in the neonatal transition period, the technical superiority of FD-NIRS devices does not outweigh the usability of the more clinically viable and widely used CW-NIRS devices. Additional haemoglobin concentration measurements may, in a research setting, provide more information on changes in cerebral haemodynamics. The absolute differences found may not be relevant in clinical practice, as trend monitoring of $\mathrm{rcSO}_{2}$ in combination with $\mathrm{SpO}_{2}$ may suffice to guide support during the transition.

\section{Supplementary information}

Supplementary information accompanies this paper at https://doi.org/10. 1186/s12887-020-1987-4.

Additional file 1. Table SI: Phase shift of the patients measured with FD-NIRS

\begin{abstract}
Abbreviations
AC: Amplitude of modulation; CW: Continuous-wave; DC: Average intensity; FD: Frequency-domain; HHb: Deoxygenated haemoglobin concentration; HR: Heart rate; NIRS: Near-infrared spectroscopy; $\mathrm{O}_{2} \mathrm{Hb}$ : Oxygenated haemoglobin concentration; PEEP: Positive end-expiratory pressure; $\mathrm{rcSO}_{2}$ : Regional cerebral oxygen saturation; $\mathrm{SpO}_{2}$ : Arterial oxygen saturation; THb: Total haemoglobin concentration
\end{abstract}

\section{Acknowledgements \\ We thank Ko Hagoort for providing editorial advice and Katya Mauff for providing statistical advice.}

\section{Authors' contributions}

TG and RJ designed the study. TE and LB performed the measurements. GP and BU provided original data from the Medical University of Graz. TE processed the data, performed the analysis, drafted the manuscript and designed the Figs. TG and RJ aided in interpreting the results and worked on the manuscript. RJ and IR were involved in planning and supervising the work. All authors discussed the results and commented on the manuscript. 


\section{Funding}

Not applicable.

\section{Availability of data and materials}

The data that support the findings of this study are available from Erasmus MC - Sophia Children's Hospital, but restrictions apply to the availability of data from the Medical University of Graz, which were used under licence for the current study, and so are not publicly available. Data are, however, available from the authors upon reasonable request and with permission from the Medical University of Graz.

\section{Ethics approval and consent to participate}

The Research Ethics Board at Erasmus Medical Center waived the requirement to obtain informed consent for data gathered at the Erasmus Medical Center Sophia Children's Hospital. (argument: "Medical Research in Human Subjects Act does not apply to this research proposal"; MEC-2011-415).

The gathering of data was approved by the Regional Committee on Biomedical Research Ethics at the Medical University of Graz (EK- number: 19-291 ex07/08, 23-403ex10/11, 27-465ex14/15). Written informed consent was obtained from the parents before the birth of the infant.

\section{Consent for publication}

Not applicable.

\section{Competing interests}

The authors declare that they have no competing interests.

\section{Author details}

'Department of Pediatrics, Division of Neonatology, Erasmus MC - Sophia Children's Hospital, Dr. Molewaterplein 40, 3015GD Rotterdam, The Netherlands. ${ }^{2}$ Department of Biomechanical Engineering, Delft University of Technology, Mekelweg 5, 2628CD Delft, The Netherlands. ${ }^{3}$ Research Unit for Neonatal Micro- and Macrocirculation, Department of Pediatrics and Adolescent Medicine, Medical University of Graz, Auenbruggerplatz 2, 8036 Graz, Austria. ${ }^{4}$ Division of Neonatology, Department of Pediatrics and Adolescent Medicine, Medical University of Graz, Auenbruggerplatz 2, 8036 Graz, Austria. ${ }^{5}$ Pediatric Intensive Care Unit, Department of Pediatrics and Pediatric Surgery, Erasmus MC - Sophia Children's Hospital, Dr. Molewaterplein 40, 3015GD Rotterdam, The Netherlands.

Received: 5 April 2019 Accepted: 18 February 2020 Published online: 28 February 2020

\section{References}

1. Pansy J, Baik N, Schwaberger B, Scheuchenegger A, Pichler-Stachl E, Avian $A$, et al. Cerebral hypoxia during immediate transition after birth and short term neurological outcome. Early Hum Dev. 2017;110:13-5.

2. Verhagen EA, Van Braeckel KN, van der Veere $\mathrm{CN}$, Groen $\mathrm{H}$, Dijk PH, Hulzebos CV, et al. Cerebral oxygenation is associated with neurodevelopmental outcome of preterm children at age 2 to 3 years. Dev Med Child Neurol. 2015;57(5):449-55.

3. Viaroli F, Cheung PY, O'Reilly M, Polglase GR, Pichler G, Schmolzer GM. Reducing Brain Injury of Preterm Infants in the Delivery Room. Front Pediatr. 2018;6:290.

4. Garvey AA, Kooi EMW, Smith A, Dempsey EM. Interpretation of Cerebral Oxygenation Changes in the Preterm Infant. Child (Basel). 2018;5(7).

5. Schneider A, Minnich B, Hofstatter E, Weisser C, Hattinger-Jurgenssen E, Wald M. Comparison of four near-infrared spectroscopy devices shows that they are only suitable for monitoring cerebral oxygenation trends in preterm infants. Acta Paediatr. 2014;103(9):934-8.

6. Hessel TW, Hyttel-Sorensen S, Greisen G. Cerebral oxygenation after birth - a comparison of INVOS((R)) and FORE-SIGHT near-infrared spectroscopy oximeters. Acta Paediatr. 2014;103(5):488-93.

7. Binder C, Urlesberger B, Avian A, Pocivalnik M, Muller W, Pichler G. Cerebral and peripheral regional oxygen saturation during postnatal transition in preterm neonates. J Pediatr. 2013;163(2):394-9.

8. Pichler G, Binder C, Avian A, Beckenbach E, Schmolzer GM, Urlesberger B. Reference ranges for regional cerebral tissue oxygen saturation and fractional oxygen extraction in neonates during immediate transition after birth. J Pediatr. 2013;163(6):1558-63.

9. R Core Team. R: A language and environment for statistical computing. R Foundation for Statistical Computing. Vienna, Austria2017.
10. Fenton TR, Kim JH. A systematic review and meta-analysis to revise the Fenton growth chart for preterm infants. BMC Pediatr. 2013;13:59.

11. Pichler G, Baik N, Urlesberger B, Cheung PY, Aziz K, Avian A, et al. Cord clamping time in spontaneously breathing preterm neonates in the first minutes after birth: impact on cerebral oxygenation - a prospective observational study. J Matern Fetal Neonatal Med. 2016;29(10):1570-2.

12. Popat H, Galea C, Evans N, Lingwood B, Colditz PB, Halliday R, et al. Effect of delayed cord clamping on cerebral oxygenation in very preterm infants. Neonatology. 2019;115(1):13-20.

13. Dix LM, van Bel F, Baerts W, Lemmers PM. Comparing near-infrared spectroscopy devices and their sensors for monitoring regional cerebral oxygen saturation in the neonate. Pediatr Res. 2013;74(5):557-63.

14. Pocivalnik M, Pichler G, Zotter H, Tax N, Muller W, Urlesberger B. Regional tissue oxygen saturation: comparability and reproducibility of different devices. J Biomed Opt. 2011;16(5):057004.

15. Szczapa T, Karpinski L, Moczko J, Weindling M, Kornacka A, Wroblewska K, et al. Comparison of cerebral tissue oxygenation values in full term and preterm newborns by the simultaneous use of two near-infrared spectroscopy devices: an absolute and a relative trending oximeter. J Biomed Opt. 2013;18(8):87006.

16. Davies DJ, Clancy M, Lighter D, Balanos GM, Lucas SJE, Dehghani H, et al. Frequency-domain vs continuous-wave near-infrared spectroscopy devices: a comparison of clinically viable monitors in controlled hypoxia. J Clin Monit Comput. 2017;31(5):967-74.

17. Dullenkopf A, Kolarova A, Schulz G, Frey B, Baenziger O, Weiss M. Reproducibility of cerebral oxygenation measurement in neonates and infants in the clinical setting using the NIRO 300 oximeter. Pediatr Crit Care Med. 2005;6(3):344-7.

18. Menke J, Voss U, Moller G, Jorch G. Reproducibility of cerebral near infrared spectroscopy in neonates. Biol Neonate. 2003;83(1):6-11.

19. Sorensen LC, Greisen G. Precision of measurement of cerebral tissue oxygenation index using near-infrared spectroscopy in preterm neonates. J Biomed Opt. 2006;11(5):054005

20. Scheeren TW, Schober $P$, Schwarte LA. Monitoring tissue oxygenation by near infrared spectroscopy (NIRS): background and current applications. J Clin Monit Comput. 2012;26(4):279-87.

21. Franceschini MA, Thaker $\mathrm{S}$, Themelis G, Krishnamoorthy KK, Bortfeld H, Diamond SG, et al. Assessment of infant brain development with frequencydomain near-infrared spectroscopy. Pediatr Res. 2007;61(5 Pt 1):546-51.

22. Zhao J, Ding HS, Hou XL, Zhou CL, Chance B. In vivo determination of the optical properties of infant brain using frequency-domain near-infrared spectroscopy. J Biomed Opt. 2005;10(2):024028.

23. Pichler G, Wolf M, Roll C, Weindling MA, Greisen G, Wardle SP, et al. Recommendations to increase the validity and comparability of peripheral measurements by near infrared spectroscopy in neonates. 'Round table', section of haematology, oxygen transport and microcirculation, 48th annual meeting of ESPR, Prague 2007. Neonatology. 2008;94(4):320-2.

24. Kleiser S, Nasseri N, Andresen B, Greisen G, Wolf M. Comparison of tissue oximeters on a liquid phantom with adjustable optical properties. Biomed Opt Express. 2016;7(8):2973-92

25. Kleiser S, Ostojic D, Andresen B, Nasseri N, Isler H, Scholkmann F, et al. Comparison of tissue oximeters on a liquid phantom with adjustable optical properties: an extension. Biomed Opt Express. 2018;9(1):86-101.

26. Schwaberger B, Pichler G, Binder C, Avian A, Pocivalnik M, Urlesberger B. Even mild respiratory distress alters tissue oxygenation significantly in preterm infants during neonatal transition. Physiol Meas. 2014;35(10):2085-99.

27. Schwaberger B, Pichler G, Binder-Heschl C, Baik N, Avian A, Urlesberger B. Transitional changes in cerebral blood volume at birth. Neonatology. 2015;108(4):253-8.

28. Arri SJ, Muehlemann T, Biallas M, Bucher HU, Wolf M. Precision of cerebral oxygenation and hemoglobin concentration measurements in neonates measured by near-infrared spectroscopy. J Biomed Opt. 2011;16(4):047005.

29. Baik N, Urlesberger B, Schwaberger B, Schmolzer GM, Mileder L, Avian A, et al. Reference ranges for cerebral tissue oxygen saturation index in term neonates during immediate neonatal transition after birth. Neonatology. 2015;108(4):283-6.

30. Urlesberger B, Kratky E, Rehak T, Pocivalnik M, Avian A, Czihak J, et al. Regional oxygen saturation of the brain during birth transition of term infants: comparison between elective cesarean and vaginal deliveries. J Pediatr. 2011;159(3):404-8.

\section{Publisher's Note}

Springer Nature remains neutral with regard to jurisdictional claims in published maps and institutional affiliations. 